\title{
Essais
}

Revue interdisciplinaire d'Humanités

Hors-série 1 | 2013

L'estrangement

\section{Le côté Šklovskij des Essais de Montaigne : l' estrangement des langues et des disciplines}

Isabelle Poulin

\section{OpenEdition}

1 Journals

Édition électronique

URL : http://journals.openedition.org/essais/2492

DOI : 10.4000/essais. 2492

ISSN : 2276-0970

Éditeur

École doctorale Montaigne Humanités

Édition imprimée

Date de publication : 15 octobre 2013

Pagination : 181-190

ISBN : 978-2-9544269-1-4

ISSN : 2417-4211

Référence électronique

Isabelle Poulin, "Le côté Šklovskij des Essais de Montaigne : l'estrangement des langues et des disciplines », Essais [En ligne], Hors-série 1 | 2013, mis en ligne le 05 octobre 2020, consulté le 12 octobre 2020. URL : http://journals.openedition.org/essais/2492 ; DOI : https://doi.org/10.4000/ essais.2492 


\title{
Le côté Šklovskij des Essais de Montaigne : l'estrangement des langues et des disciplines
}

\author{
Isabelle Poulin
}

"La critique est affaire de distance convenable. Elle est chez elle dans un monde où ce sont les perspectives et les optiques qui comptent et où il est encore possible d'adopter un point de vue."

Walter Benjamin, "Ces espaces sont à louer ". ${ }^{1}$

On connaît l'hypothèse énoncée par le narrateur de la Recherche du temps perdu : il y aurait un "côté Dostö̈evski des Lettres de Madame de Sévigné "². Pour l'avoir essayée à plusieurs reprises ${ }^{3}$, on peut affirmer que l'étrange formule proustienne fonde, dans le champ de la littérature comparée, ce que Françoise Lavocat appelle une " herméneutique de la défamiliarisation »:

"J'ai privilégié la défamiliarisation par rapport aux deux autres façons de concevoir et de pratiquer le comparatisme : l'exhumation et l'actualisation. La première [...] consiste à restituer les œuvres dans leur contexte précis et à dégager leur signification originelle [...] Par "l'actualisation", au contraire, le chercheur lit les textes anciens à travers ses propres intérêts et à la lumière de problématiques contemporaines. La défamiliarisation me parait une voix moyenne, proprement herméneutique, par laquelle est prise en considération la situation historique du chercheur mais où l'ambition est moins de restituer le sens originel et ultime d'une œuvre que de la faire voir autrement, grâce au décentrement de la perspective opéré par sa mise en relation avec d'autres œuvres et d'autres cultures. L'objectif de cette opération est la production de connaissance $[\ldots]^{4} »$.

1 Sens unique, traduit de l'allemand par Jean Lacoste, Paris, Maurice Nadeau, 2007, p. 205.

2 Marcel Proust, $\grave{A}$ l'ombre des jeunes filles en fleurs in $\grave{A}$ la Recherche du temps perdu, Paris, Gallimard, édition de la Pléiade dirigée par Jean-Yves Tadié, t. II, 1988, p. 14.

3 Dès le premier travail de recherche consacré aux « Paysages et personnages dans les œuvres de Dostoïevski et $\mathrm{M}^{\mathrm{me}}$ de Sévigné » (mémoire de Maîtrise, 1986), puis à l'occasion d'une réflexion sur les Écritures du ressassement ("Le côté Dostoïevski des Lettres Cent ans (de solitude) après ", Modernités, $\mathrm{n}^{\circ}$ 15, PUB, 2001).

4 "Le comparatisme comme herméneutique de la défamiliarisation", article mis en ligne sur le site Vox poetica en avril 2012 (http://www.vox-poetica.org/t/articles/lavocat2012.html). 
Une même méthode ayant été adoptée par un grand historien italien, Carlo Ginzburg, on s'intéressera ici à la "production de connaissance " résultant de cette "voix moyenne " du comparatisme, dans le but d'en déduire peut-être des spécificités disciplinaires, d'une langue à l'autre.

Dans l'essai intitulé en français « L'estrangement. Préhistoire d'un procédé littéraire " Lettres de Madame de Sévigné ", qu'il met en perspective avec certains écrits de Marc Aurèle et avec ceux des formalistes russes. Le souci qu'il exprime néanmoins de ne pas brouiller « les frontières entre l'histoire et la fiction " ${ }^{6}$ fait loupe sur la notion même de "procédé littéraire ». Plusieurs affirmations de Carlo Ginzburg semblent en faire un outil qu'auraient en partage les écrivains et les historiens : il est "fasciné ", dit-il, "par la façon dont des dispositifs fictionnels peuvent être employés dans des buts historiographiques " ${ }^{7}$, et il a souvent confié avoir d'abord pensé entreprendre des études de littérature ${ }^{8}$. À la fin de son article sur "L'estrangement ", il décrit " le projet historiographique dans lequel [il] se reconna[ît] personnellement » en reprenant une phrase de Proust : «Â supposer que l'histoire soit scientifique, encore faudrait-il la peindre comme Elstir peignait la mer, par l'autre sens " ${ }^{9}$. Toute une série de réserves incitent toutefois à remettre sur le métier la question de la spécificité « littéraire » - celle-là même qui hantait les formalistes russes, parmi lesquels Viktor Šklovskij, pour qui l'art tout entier était un " procédé » d'estrangement (selon la traduction française de la traduction italienne du mot russe ostranenie).

On s'interrogera tout d'abord sur le sens de cette " préhistoire " d'un procédé annoncée par le titre de l'essai. Carlo Ginzburg trouve trace de l'estrangement dans "les réflexions écrites en langue grecque au $\mathrm{II}^{\mathrm{e}}$ siècle après Jésus-Christ par l'empereur romain Marc Aurèle ${ }^{10}$ : le repérage d'un " procédé littéraire » dans une œuvre ancienne est-il une condition préalable à sa reprise par un historien ? Le " côté Šklovskij des Essais de Montaigne " voudrait signaler le moins grand recul éprouvé en traduction, l'italien "straniamento " étant restitué par un terme français vieilli qui renvoie à cet autre point de départ : la langue de Montaigne (même si celui-ci n’utilise pas le terme d' " estrangement " mais celui d'" estrangeté "; en Moyen français on

5 À distance. Neuf essais sur le point de vue en histoire, traduit de l'italien par Pierre-Antoine Fabre, NRF/Gallimard, 2001, p. 15-36.

6 «L'estrangement. Préhistoire d'un procédé littéraire », ibid., p. 36.

7 Carlo Ginzburg, entretien réalisé par Philippe Mangeot, « de près de loin », Vacarme, 18, 2002 (http://www.vacarme.org/article235.html).

8 "Vers le milieu des années cinquante je lisais des romans ; l'idée que je pourrais devenir historien ne m'effleurait même pas », Carlo Ginzburg, " Préface » de Mythes emblèmes traces, traduit de l'italien par Monique Aymard, Christian Paolini, Elsa Bonan et Martine Sancini-Vignet, Paris, Verdier/Poche, 1986, p. 9.

9 "L'estrangement ", op. cit., p. 36.

10 Ibid., p. 18. 
utilise encore " estrangement " au sens d'" aliénation : le fait d'être obligé de partir, de s'expatrier »). D'une langue à l'autre se constitue un réseau de sens (qui n'est pas une "préhistoire »), où l'on lira une première spécificité (d'une approche littéraire) de la « littérature ".

La lecture des réflexions de Marc Aurèle n'en est pas moins extrêmement troublante par ailleurs, du fait même de l'effet de modernité produit par telle représentation de l'accouplement, par exemple : «C'est le frottement d'un boyau et une sécrétion de liquide gluant accompagnée d'un spasme ${ }^{11}$. Carlo Ginzburg déplore le " désintérêt pour l'histoire, caractéristique de l'attitude des formalistes russes ${ }^{12}$ auxquels il emprunte le procédé de l'estrangement. Cette caractéristique a été nuancée, notamment à la lumière des travaux de Iouri Tynianov, qui se pose précisément la question de l'évolution des formes littéraires ${ }^{13}$. Mais on retiendra surtout ce brouillage temporel provoqué par la lecture de Marc Aurèle : " cet extraordinaire fragment apparait aux yeux d'un lecteur du $\mathrm{XX}^{\mathrm{e}}$ siècle comme un précoce exemple d'estrangement $»^{14}$ écrit Carlo Ginzburg. Est-ce cette avance sur son temps qui fait la spécificité de la littérature?

\section{Les chemins de la connaissance}

Dans le texte programmatique d'une revue pluridisciplinaire jamais réalisée mais projetée, entre autres, avec Carlo Ginzburg, Italo Calvino souligne la fonction de lieu commun qu'il semble légitime de prêter au texte littéraire :

«C'est la littérature [...] le champ qui soutient et motive cette rencontre et cette confrontation des recherches et des opérations dans des disciplines différentes, même si elles sont apparemment distantes ou étrangères. C'est la littérature comme espace de significations et de formes qui ne valent pas seulement pour la littérature. Nous croyons que les poétiques littéraires peuvent renvoyer à une poétique du faire, et même : du se faire $»^{15}$.

Le champ investi dans l'essai sur «L'estrangement » est celui de la littérature. Son point de départ est un article de Viktor Šklovskij, l'un des membres de l'OPOIAZ, abréviation russe de "Société d'étude du langage poétique " (groupe plus connu sous le terme péjoratif de "formalistes russes »). Cet article date de 1917 et s’intitule "L'art comme procédé ${ }^{16}$. Šklovskij y définit le procédé

11 Ibid., p. 20.

12 Ibid., p. 18.

13 Voir Iouri Tynianov, Formalisme et histoire littéraire, traduit du russe par Catherine DeprettoGenty, Lausanne, L'Âge d'Homme, 1991.

14 «L'estrangement ", op. cit., p. 20.

15 "Le regard de l'archéologue " (1972), Défis aux labyrinthes, I, traduit de l'italien par JeanPaul Manganaro et Michel Orcel, Paris, Seuil, 2003, p. 290-291.

16 Traduction française de Tzvetan Todorov, Théorie de la littérature. Textes des formalistes russes, Paris, Seuil, 1965. 
d' « ostranenie des choses » qui, associé à ce qu'il appelle « la complication des formes " (par où s'introduit la dimension historique de cette poétique), constitue le propre de l'art qui est selon lui « de nous procurer une sensation de la chose, mais une sensation qui soit une vision, et non pas seulement une reconnaissance ${ }^{17}$. Voir l'accouplement et non le reconnaître, revient par exemple à le définir comme "le frottement d'un boyau et une sécrétion de liquide gluant accompagnée d'un spasme " (Marc Aurèle). C'est une question de regard, que souligne clairement le titre original du livre de Carlo Ginzburg : Occhiacci di legno. Nove riflessioni sulla distanza, par sa référence aux "gros yeux de bois " de Pinocchio (Collodi, Le avventure di Pinocchio, 1881).

Ce regard, la référence à la lecture de Collodi l'atteste, est tout à la fois entretenu et soutenu par la langue. L'examen de quelques traductions suffit à souligner combien le lien entre le "stranamiento » et l'étrangeté d'un regard nouveau (celui de Pinocchio) est peu assuré, d'une langue à l'autre. Il disparait presque en français : $\grave{A}$ distance. Neuf essais sur le point de vue en histoire. Il semble instable en anglais : le "Wooden eyes » du titre de l'essai (Wooden Eyes: Nine Reflections on Distance) ne coïncide pas avec les traductions usuelles de la scène du chapitre III à laquelle puise Carlo Ginzburg ("Occhiacci di legno, perché mi guardate? " est traduit tantôt par "Wicked [mauvais] wooden eyes ", tantôt par "Ugly [horrible] wooden eyes »). On peut préciser par ailleurs que le mot russe ostranenie, traduit en italien par stranamiento, a connu maintes versions en français qui insistent tantôt sur une inquiétante étrangeté (" étrangisation " ${ }^{18}$, " défamiliarisation "), tantôt sur l'étonnement ("singularisation », " aliénisation»), tantôt sur l'éloignement, en référence à la place ou à la formation du lecteur / spectateur ( distanciation $»^{19}$ ).

17 Cité par Carlo Ginzburg in "L'estrangement ", op. cit., p. 16.

18 C'est l'entrée du Dictionnaire international des termes littéraires (J. M. Grassin) ; voir DITL en ligne : http://www.ditl.info/arttest/art76.php, article "Étrangisation : défamiliarisation/ ostranenie ». Le terme d' "étrangisation » est attesté dans la traduction par Michel Pétris de $\mathrm{La}$ Marche du cheval du même Šklovskij, Paris, Champs libre, 1973.

19 C'est la notion par laquelle sont passés les traducteurs français, comme l'indique la précision accompagnant "L'estrangement. Préhistoire d'un procédé littéraire " : "Nous choisissons de traduire l'italien straniamento (d'après le russe ostranenie) par ce terme d'Antoine Vitez dans un remarquable texte sur la distanciation [Verfremdung] brechtienne (in De Chaillot à Chaillot, Paris, 1981, p. 57) ", NdT, p. 15. On trouve par ailleurs in le Dictionnaire des termes littéraires de H. van Gorp, D. Delabastita, L. D’hulst, R. Ghesquiere, R. Grutman et G. Legros (Champion Classiques, 2005), l'entrée suivante : " Distanciation (effet de -) (angl.alienation effect [the distancing effect, more commonly known (earlier) by John Willett's, 1964, translation the alienation effect or (more recently) as the estrangement effect]; all. Verfremdungseffekt). Procédé théâtral introduit par B. Brecht afin de perturber la traditionnelle illusion dramatique. Brecht voulait orienter l'énergie ainsi libérée chez le spectateur vers le contenu doctrinal de la pièce et vers ses réflexions à portée sociale. Il crée l'effet de distanciation en rendant inhabituel ce qui va normalement de soi, démarche qui fait accéder le spectateur à une compréhension d'autant meilleure de la pièce (cf. l'"ostranenie" [désautomatisation ou singularisation] du Formalisme russe)». 
Ces hésitations nous conduisent là où s'arrête la traduction et qui s'appelle l'histoire : pour qui n'a pas eu Pinocchio dans son enfance les "yeux de bois" (et toutes les nuances construites par l'histoire de Collodi) ne réveillent rien, ne construisent aucune perspective de pensée. Le terme français d'estrangement choisi par les traducteurs renvoie plutôt à Montaigne, pas à Collodi. Il met en branle d'autres circuits imaginaires. Qu'en déduire? "Quelque langue que parlent mes livres, je leur parle en la mienne", affirmait l'auteur des Essais ${ }^{20}$. Il ne s'agit pas de prôner par là le moindre relativisme. Les circuits empruntés par les langues peuvent être différents et conduire à un même point de vue. C'est, semble-t-il, ce que cherche à mettre en évidence Carlo Ginzburg dans son article en repérant, dans des temps et des écritures très différentes, un même "procédé littéraire ", précisément parce que la littérature s'offre comme cet espace de traduction (au sens où l'entend Proust : " La tâche et le devoir d'un écrivain sont ceux d'un traducteur ${ }^{21}$ ) où se construisent des unités de signification plus grandes que les mots, plus mobiles que les concepts, et susceptibles de coïncidence, favorisant la construction de " comparables".

Revenons sur le cheminement de lecture de Carlo Ginzburg et sa quête d'une "préhistoire ». L'historien part de Šklovskij et de la lecture de Tolstoï faite par le critique russe. Il remonte à Marc Aurèle (qu'avait lu Tolstoï), passe par les Lumières (et des écrivains, Voltaire, La Bruyère, qu'avait lus Tolstoï). Retrouvant chez les uns et les autres un même "procédé " de mise à nu de la réalité, il met ainsi en évidence le débordement permanent des frontières propre aux écrivains - qui se lisent les uns les autres, et se comprennent même dans de mauvaises traductions linguistiques (comme ce fut le cas de Proust, l'un des plus subtils lecteurs, en son temps, de l'œuvre de Dostoïevski pourtant sévèrement mutilée par les premières traductions françaises).

De Šklovskij à Proust, Carlo Ginzburg n'établit pas de rapport de fait, mais souligne une coïncidence de dates : le premier forge son procédé entre 1917 et 1922 ; le second publie Du côté de chez Swann en 1917 et À l'ombre des jeunes filles en fleurs, qui contient l'allusion au " côté Dostoïevski des Lettres de Madame de Sévigné ", en 1918. Cette contemporanéité permet l'hypothèse d'un écho : "L'idée [de Šklovskij] selon laquelle l'art serait un instrument pour raviver notre perception figée par l'habitude rappelle immédiatement la fonction attachée à la mémoire involontaire dans l'œuvre de Marcel Proust $»^{22}$. Certes, la mémoire involontaire entraîne d'abord une refamiliarisation (avec son passé), mais dans la mesure où elle est aussi une actualisation du passé donnant à voir le présent, elle participe de la même esthétique proustienne

20 "Des livres », Les Essais, livre second, chapitreX, édition réalisée par Denis Bjaï, Bénédicte Boudou, Jean Céard et Isabelle Pantin, Paris, Le Livre de Poche, Classique, 2002, p. 141.

21 Le Temps retrouvé in $A$ la Recherche du temps perdu, op. cit., t. IV, 1989, p. 469.

22 Carlo Ginzburg, "L'estrangement ", op. cit., p. 16. 
que " le côté Dostö̈evski des Lettres de Madame de Sévigné » : on ne connaît, "souvent longtemps après, la beauté d'une chose que dans une autre " ${ }^{23}$.

Carlo Ginzburg pourrait entretenir d'autant plus fermement ce parallèle que Proust avait lu Tolstoï (qui avait lu La Bruyère, Voltaire et Marc Aurèle). "Le côté Dostoïevski des Lettres de Madame de Sévigné » ne lui semble d'ailleurs pas " une notion (très) différente de l'estrangement ». Une réserve s'introduit toutefois à ce point de l'analyse, où s'aperçoit une spécificité disciplinaire. Ce qui intéresse Carlo Ginzburg, ce sont " les implications épistémologiques » de l'estrangement ; or Proust ne les dévoile, selon lui, qu' « en déplaçant son intérêt pour les tableaux [d'Elstir, troisième terme de la comparaison proustienne] et les romans sur le terrain de la meilleure analyse possible d'un grand événement ${ }^{24}$ :

«À supposer que la guerre soit scientifique, encore faudrait-il la peindre comme Elstir peignait la mer, par l'autre sens, et partir des illusions, des croyances qu'on rectifie peu à peu, comme Dostoïevski raconterait une vie $»^{25}$.

Dans une étude de 1987 consacrée à ce même " côté Dostoïevski », le philosophe Vincent Descombes refusait d'y croire, arguant que " $\mathrm{M}^{\mathrm{me}}$ de Sévigné et Elstir représentent les apparences en tant qu'illusions d'optique, tandis que Dostoïesvki les représente en tant qu'elles passent pour la vérité »- et la vérité vraie "demande le Temps" pour se révéler, ce qui est le privilège du roman, précisait-il, mais ni des tableaux impressionnistes ni des descriptions de paysages faites par la Marquise ${ }^{26}$. Ce " côté " existe bel et bien, pourtant, en tant que procédé cognitif, qui ne consiste pas à dire que les arts poétiques sont les mêmes, mais qu'une lecture "à distance " en ravive les pouvoirs propres : le pas de lecture, si caractéristique, de Carlo Ginzburg, en est une preuve éclatante. On peut se demander néanmoins pourquoi les « implications épistémologiques » de l'estrangement ne seraient perceptibles qu'à la lampe d'un "grand événement »? Lorsqu'il se fait critique d'art (de tableaux, de romans ou de Lettres), Proust ne dévoile-t-il aucune "implication épistémologique " ? Répondre par l'affirmative revient à préciser la nature et la portée de cette "production de connaissance » spécifique à l'écrivain, auquel l'historien emprunte la petite échelle de son champ de recherche, sans suivre toutefois les mêmes traces.

\section{L'empreinte de la langue}

La lecture de Marc Aurèle produit un effet de familiarité qui comporte un risque d'anachronisme, ou d'achronisme. Or pour Šklovskij ostranenie est un néologisme. Il marque un (re)commencement, ou en tout cas il lui permet

23 Marcel Proust, Le Temps retrouvé, op. cit., p. 560.

24 "L'estrangement", op. cit., p. 35, je souligne.

25 Marcel Proust, Le Temps retrouvé, cité par Carlo Ginzburg, "L'estrangement ", ibid.

26 Vincent Descombes, Proust, Philosophie du roman, Paris, éditions de Minuit, 1987, p. 267. 
de se démarquer des critiques littéraires qui l'ont précédé : sa définition de l'art comme ce qui sert à renforcer les perceptions, veut mettre en défaut la position de Potebnia pour lequel l'image, en art, sert à penser. Par ailleurs, Śklovskij applique ce terme à la littérature russe : pourquoi celle-ci et pas une autre, se demande Carlo Ginzburg ? On peut lire une ébauche de réponse dans une précision donnée par le critique russe : "La langue littéraire russe [...] est d'une origine étrangère pour la Russie $»^{27}$. C'est un certain usage de l'étranger, daté (la " littérature russe » s'édifie au XIX siècle), qui rend perceptible le procédé de "l'étrangement ", dont on peut donc dire qu'il repose sur la capacité à inventer de nouveaux points de départ.

Marc Aurèle sert à " penser ailleurs ", comme la ville de Los Angeles, où s'imposent clairement à Carlo Ginzburg les vertus de la distance ${ }^{28}$.

Pensons ailleurs est le titre d'un livre de la sociologue Nicole Lapierre, emprunté à Montaigne, qui s'intéresse aux intellectuels qui s'en sont allés penser ailleurs, franchissant les frontières réelles et les barrières sociales : George Simmel, Edward Said, Walter Benjamin ou Gilles Deleuze. Elle veut montrer que l'intellectuel critique est toujours une personne déplacée, parfois au sens propre, "mais toujours au sens figuré $»^{29}$.

L'emprunt à Montaigne vient du livre III des Essais, du chapitre IV, intitulé "De la diversion ", qui s'ouvre sur les chagrins et les deuils, l'art difficile de la consolation. L'écrivain donne en suivant des exemples de situations critiques (celle d'un condamné à mort sur l'échafaud, par exemple) auxquelles l'homme cherche à échapper en pensant à autre chose :

«Nous pensons toujours ailleurs : l'espérance d'une meilleure vie nous arrête et appuie : ou l'espérance de la valeur de nos enfants : ou la gloire future de notre nom ; ou la fuite des maux de cette vie : ou la vengeance qui menace ceux qui nous causent la mort $»^{30}$.

L'expression peut sembler métaphorique seulement, mais Montaigne précise à propos de l'" aigre imagination " qui tient quiconque se met en quête de "véhémente diversion":

«Si je ne puis la combattre, je lui échappe : et en la fuyant, je fourvoie, je ruse :

Muant de lieu, d'occupation, de compagnie, je me sauve dans la presse d'autres amusements et pensées, où elle perd ma trace et m'égare " ${ }^{31}$.

On trouve par ailleurs, sous la plume de Montaigne, l'expression « Un homme qui pense ailleurs » dans le livre II, chapitre XVII, intitulé « De la présomption ». Il s'agit là de mettre en évidence les effets de l'étranger sur les habitudes :

27 Viktor Šklovskij, "L'art comme procédé ", Théorie de la littérature, op. cit., p. 96.

28 Voir « Préface", À Distance, op. cit., p. 11-13.

29 Nicole Lapierre, Pensons ailleurs, Paris, Stock, Folio/Essais, 2004, p. 28.

30 Essais, livre III, "De la diversion », Le Livre de poche, op. cit., p. 83.

31 Ibid., p. 85-86; je souligne. 
"Autrefois étant en lieu, où c'est discourtoisie barbaresque de ne répondre à ceux qui vous convient à boire : quoi qu'on m'y traitât avec toute liberté, j'essayai de faire le bon compagnon en faveur des dames qui étaient de la partie, selon l'usage du pays. Mais il y eut du plaisir : car cette menace et préparation d'avoir à m'efforcer outre ma coutume et mon naturel, m'estoupa de manière le gosier, que je ne sus avaler une seule goutte : et fut privé de boire pour le besoin même de mon repas. Je me trouvai saoul et désaltéré par tant de breuvage que mon imagination avait préoccupé. [...] Un homme qui pense ailleurs ne faudra point, à un pousse près, de refaire toujours un même nombre de pas au lieu où il se promène : mais s'il y est avec attention de les mesurer et conter, il trouvera que ce qu'il faisait par nature et par hasard, il ne le fera pas si exactement par dessein ${ }^{32}$.

On voit bien que la " diversion » dont il est question chez Montaigne n'est pas si éloignée de l'estrangement (ostranenie) : elle désigne cet écart qui permet une vision (de soi, du monde) et non une reconnaissance (parfois source de confusion, certes, comme le souligne le dernier exemple dans lequel se voir faire, revient à ne plus rien savoir). Or l'auteur des Essais lie expressément les difficultés qu'un homme éprouve à sortir de lui-même à de tout petits rouages :

"Peu de chose nous divertit et détourne : car peu de chose nous tient. Nous ne regardons guère les sujets en gros et seuls : ce sont des circonstances ou des images menues et superficielles, qui nous frappent : et des vaines écorces qui rejaillissent des sujets.

\section{Folliculos ut nunc teretos aestate cicadae} Linquunt $»^{33}$.

La citation de Lucrèce : "Comme, l'été venu, les cigales abandonnent leurs rondes membranes ", participe doublement de ce mouvement d'estrangement qui caractérise la poétique de Montaigne : elle est en langue étrangère, et met en évidence cette petite échelle (des peaux déliées des cigales) à laquelle travaille tout écrivain aspirant à déprendre les hommes de leurs automatismes. C'est l'échelle des corps et des langues, qui produit une connaissance que l'on peut qualifier de "vérité poétique ". Elle concerne la façon dont "peu de chose nous tient ", mais nous tient ferme : ce qui s'appelle imagination, et qu'entretient l'imaginaire d'une langue. La fonction du poète (de tout étrangeur de langue, si l'on nous permet ce néologisme) est de mettre à distance un imaginaire reçu. Il sait comment s'entre-tiennent les êtres de langage, et c'est ce savoir qui intéresse la critique d'art présente dans la Recherche proustienne. Il peut faire l'effet d'une prescience (c'est le caractère " précoce » du fragment de Marc Aurèle) parce qu’il met en évidence des mécanismes microscopiques, la vie dans/de la langue, que viennent confirmer parfois, et grossir, de "grands » événements. En prenant ses distances par rapport à un certain état de la langue, le poète contribue à une forme de microhistoire du présent. Les conditions historiques de ces moments

32 Essais, livre II, « De la présomption ", Le livre de poche, op. cit., p. 497-498; je souligne.

33 Essais, livre III, «De la diversion », p. 87 ; citation du De natura rerum, V, p. 803-804. 
critiques (en ce qu'ils mettent en crise les représentations et leurs automatismes) font l'objet de recherches propres, éminemment précieuses et complémentaires, ce dont Carlo Ginzburg a fait une démonstration magistrale dans Le Fromage et les vers, en faisant entrer la catégorie de la lecture (qui sait faire quoi, et quand, d'un imaginaire reçu ?) dans son champ.

\section{Épilogue : Pinocchio, Menocchio, Seveso}

On trouve à la lecture de Carlo Ginzburg la même puissance d'estrangement que celle qu'aimait à analyser Šklovskij chez Tosltoï. Le titre de l'étude consacrée à « l'univers d'un meunier du XVI ${ }^{\mathrm{e}}$ siècle » en convainc aisément. Il reprend les paroles du meunier lui-même, Menocchio, les mots dont s'est servi ce dernier pour exposer sa "très singulière cosmogonie " : "J'ai dit que, à ce que je pensais et croyais, tout était chaos, c'est-à-dire terre, air, eau et feu tout ensemble ; et que ce volume peu à peu fit une masse, comme se fait le fromage dans le lait et

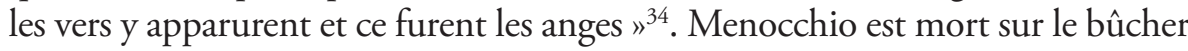
de l'Inquisition, mais les traces qu'il a laissées et dont l'historien nous permet de mesurer les effets de distanciation ont une actualité saisissante.

Elles constituent en effet, semble-t-il, le point de départ du livre récent de l'anthropologue Éric Chauvier, intitulé Somaland ${ }^{35}$. Il s'agit du compte rendu d'une enquête de terrain, menée dans l'agglomération voisine d'une zone industrielle classée "Seveso ", du nom d'une commune italienne victime en 1976 d'un rejet accidentel de Dioxine (une directive européenne du même nom, datant de 1982, demande désormais aux États et aux entreprises d'identifier les risques associés à certaines activités industrielles dangereuses et de prendre les mesures nécessaires pour y faire face). Éric Chauvier a interrogé aussi bien les habitants que les membres d'un comité local d'information regroupant les industriels ou leurs communicants, les élus, et les associations (ces comités ont été créés au lendemain de l'explosion, en septembre 2001, de l'usine AZF à Toulouse). "Somaland » est une référence au " soma ", la drogue euphorisante distribuée au travail en fin de journée dans Le Meilleur des mondes d'Aldous Huxley : elle désigne le dispositif lénifiant des réunions de ces comités. Un jeune homme résidant dans la zone dite " sensible " est persuadé, en effet, que le solvant dégagé par l'usine a altéré la santé de son amie. Or sa parole est inaudible dans un monde dominé par l'outil Power Point et son langage creux. C'est pourquoi cette parole est soutenue, dans le livre d'Éric Chauvier, par celle de Menocchio, citée à plusieurs reprises. L'auteur confirme ainsi très précisément les vertus de la distance, qui permettent d'apercevoir, dans une

34 Le Fromage et les vers, L'univers d'un meunier du XVI' siècle, traduit de l'italien par Monique Aymard, Paris, Aubier, Histoires, 1980, p. 38.

35 Paris, Allia, 2012. 
profondeur de champ retrouvée, les mécanismes d'emprise et de déprise les plus contemporains. Le chapitre consacré aux petites gens du XXI siècle s'achève sur une citation du livre de Carlo Ginzburg, dont la mise en perspective est un témoignage fort en faveur du pouvoir herméneutique de la défamiliarisation, ou du croisement des disciplines (Pinocchio, Menocchio, Seveso) :

"Qu'un meunier comme Menocchio en soit arrivé à formuler des idées aussi différentes des idées courantes sans aucune influence extérieure parut incroyable aux Inquisiteurs $~^{36}$.

Isabelle Poulin

EA 419 TELEM

Université Michel de Montaigne Bordeaux 3

Isabelle.Poulin@u-bordeaux3.fr

\title{
Résumé
}

Le croisement des disciplines auquel se livre Carlo Ginzburg dans son article «L'estrangement. Préhistoire d'un procédé littéraire ", associé au croisement des langues qu'impose la lecture en traduction française d'un historien italien confronté aux formalistes russes, invite à s'interroger sur la production de connaissance propre à une herméneutique de la défamiliarisation. Elle atteint ici tous les rouages de la pensée : la langue, l'imagination, les outils conceptuels. Paradoxalement peut-être, c'est le retour à Montaigne, favorisé par la voix traductrice (le choix du terme français estrangement pour rendre straniamento ou ostranenie), qui permet de faire l'hypothèse d'un savoir propre à l'écrivain auquel l'historien emprunte, certes, la micro-échelle de son champ de recherche, sans suivre toutefois les mêmes traces.

\section{Mots-clés}

Estrangement, ostranenie, défamiliarisation, plurilinguisme, littérature et histoire.

\begin{abstract}
Carlo Ginzburg's cross-disciplinary approach in his article "Making it strange. The Prehistory of a Literary device", combined with the interweaving of languages involved in reading the French translation of an Italian historian's writings on Russian formalism, calls for the examination of the specific production of knowledge deriving from a hermeneutic of defamiliarization. It affects all the inner workings of one's thought: language, imagination, conceptual tools. Paradoxically enough, the translating voice leads back to Montaigne (through the use of the French word estrangement to translate straniamento in the original paper), enabling us to assume that writers and historians are not following in the same footsteps.
\end{abstract}

\section{Keywords}

Estrangement effect, defamiliarization, multi-lingualism, literature and history. 